\title{
Preparative study regarding the implementa- tion of a muscular fatigue model in a virtual task simulator
}

\author{
David Brouillette $^{\mathrm{a},{ }^{*}}$, Guillaume Thivierge ${ }^{\mathrm{b}}$, Denis Marchand ${ }^{\mathrm{b}}$ and Julie Charland ${ }^{\mathrm{a}}$ \\ ${ }^{a}$ Virtual Ergonomics, Dassault Systemes, 393 St-Jacques West (suite 300), Montreal, Qc, Canada \\ ${ }^{b}$ Department of Kinesiology, Université du Québec à Montréal (UQAM), Montreal, Qc, Canada
}

\begin{abstract}
Muscle fatigue is considered as one of the major risk factors for developing musculoskeletal disorders. The aim of this project was to select an adequate fatigue assessment model for an implementation in Dassault Systemes digital human modeling software. A review of existing MET models has been done resulting in a decision to use the extended Ma's model (2010). In this project, only shoulder and elbow joints have been tested and more subjects will be necessary for further validation. The model has been compared to several endurance time (ET) static studies. Two dynamic experiments were also performed by two different subjects. The results showed that because of the inter-individual variability, a simple prediction curve or value, can't well predict individual measured ET (or task failure). There is a need for a chart representation which also shows standard deviation (SD) range. Considering the SD range, the results were included in the prediction. Thus, this range may help the human factors expert to nuance the prediction results while considering environment factors and some realities specific to the industry.
\end{abstract}

Keywords: Muscle exhaustion; Human Modeling; Digital 3d Manikin; Human Simulation

\section{Introduction}

Musculoskeletal disorder (MSD) is considered as one of the major health problems related to physical labor especially in jobs requiring manual work. The United States Department of Labor (2008) defines MSD as: cases where the nature of the injury or illness is sprains, strains, tears, or musculoskeletal system and connective tissue diseases and disorders, when the event or exposure leading to the injury or illness is bodily reaction such as twisting, overexertion and repetition. There are numerous "risk factors" associated with work-related MSDs, such as physical workload factors (Burdorf, 1992), psychosocial factors (Bongers and al., 1993) and individual factors (Armstrong and al., 1993). According to the analysis in Occupational Biomechanics (Chaffin and al., 1999), "Since muscle fatigue reduces muscle power, induces discomfort and pain, and in the long term, is believed to contribute to Cumulative Trauma Disorders (CTDs), it is important to quantify fatigue and to determine the limits of acceptable muscle loads". Muscle fatigue, for its part, has been defined as "any exercise-induced reduction in the ability to exert muscle force or power, regardless of whether or not the task can be sustained" (Bigland-Ritchie and Woods, 1984) and the "failure to continue working at a given exercise intensity" (Booth and Thomason 1991). Maximum holding or endurance time (MET) is a primary outcome variable used to quantify muscle fatigue development, particularly as a function of static contraction task intensity. In the industry, the fatigue quantification can benefit the worker by helping to adapt the work load. However, the industry needs access to adequate analysis tools. With the improvement of technologies, there are a growing

Corresponding author. Email : david.brouillette@3ds.com 
number of industries that are using digital human modeling (DHM) software. Chaffin (2001) defines DHM as: "Thus it now is possible to position and move computer generated hominoids, or avatars, as some like to call them, to predict the performance capabilities of designated groups of people within a computer rendered environment." The merge of a fatigue prediction tool and DHM software could help to detect the presence of risk of MSDs associated with fatigue level especially at an early stage of project development.

The main objective of this project was to choose an adequate fatigue model. At this point, only the shoulder and elbow have been targeted. In addition, this project is an opportunity to test one way to present the results. To achieve this work, a literature review was done in order to extract the most appropriate fatigue model. An assessment of the model implementation feasibility in the Dassault Systemes Virtual Ergonomics solution has been done through preliminary experiences in laboratory. The results of these tests will be discussed to determine if this model results corresponds to the expected value and if the results presentations could help the industry to consider and use fatigue analysis in their ergonomics intervention with DHM tools.

\section{Literature}

A large amount of static fatigue prediction curves are available in literature. However, since the intent of the current project was to find a useful and implementable model for DHM, not all existing models have been reviewed. Also, to simplify the research only the recent scientific papers and most known authors have been reviewed. The MET is generally presented graphically using either exponential $\left(\mathrm{e}^{-\mathrm{x}}\right)$ or power $\left(\mathrm{y}^{-\mathrm{x}}\right)$ curve. Some models are based on exponential function (Rohmert 1960, Sato and Al. 1984, Sjogaard 1986), but most of the MET models now use power function. As shown in figure 1 and according to Frey Law and Avin (2010), "Clearly both functions (power and exponential) predict curvilinear relationships between intensity and endurance time (ET), but the exponential model may under-predict ET at the very low task intensities"

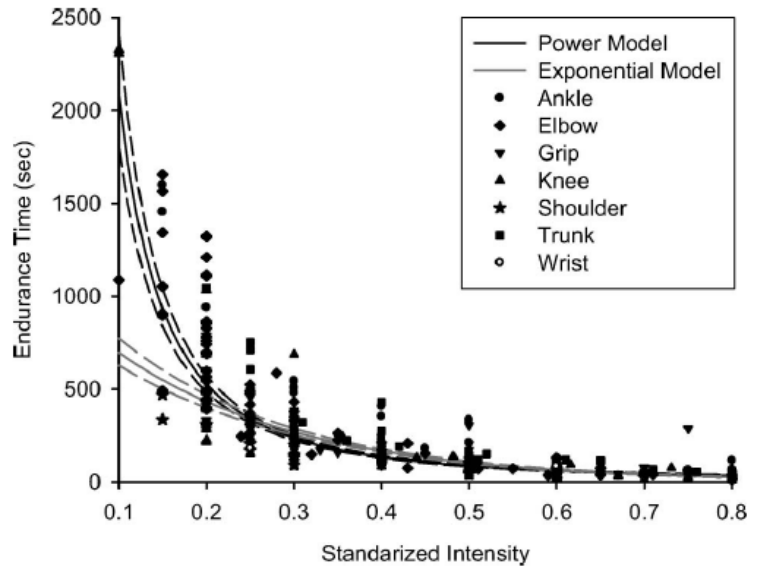

Fig. 1: General power and exponential endurance time (ET) models and specific ET extracted from several studies. Extracted from Frey Law and Avin (2010).

Rohmert's curve (Rohmert, 1960) has been the guideline regarding fatigue models for many years. However, this MET model has demonstrated a major loophole. Rohmert's MET model is an asymptotic curve at a value of $15 \%$ of maximal voluntary contraction (MVC). The term asymptote is used to describe a curve tendency towards infinite. Therefore, it assumes that any static contraction below $15 \%$ of MVC could be sustained indefinitely. This fact still cited in many ergonomics guidelines, has been refuted by many studies including Mathiassen and Ashberg (1999) and should not be used anymore by practitioners.

An article from El ahrache et al. (2005) presented a good review of the actual available literature and was used to find and review most known models. They used 24 existing MET models and grouped them in function of their relative specificity (upper limbs, back/hip and general). For each group they extracted one representative endurance time (ET) curve related to intensity of static effort. They used a percentile $\left(15^{\text {th }}\right)$ approach to avoid the inclusion of extreme values.

In 2009, Ma et al. produced a power equation to define their muscle fatigue model. They compared their equation with other models listed in El ahrache et al. (2006). Their results have shown great similarities with several previous MET models (intraclass correlation>0.90). As in El ahrache et al. (2005), the equation had to be adapted to fit the different endurance of muscle groups. Ma's model got more specific by adapting the equation to joint level. The muscle fatigue equation is extended ( $\mathrm{Ma}$ and al., 
2010) by calculating the torque of each joint, see Eq. (1) and the related parameters in Table 1. A constant (k), different for each joint, is inserted in the fatigue equation. They have determined (see Table 2) which constant $(\mathrm{k})$ value would be required to fit several existing prediction models. A mean value has been calculated from those constants to determine a value to be used for each joint. This methodology makes Ma's curve an average representation of the fatigue prediction models. The specific constants associated to other models are also used to calculate a standard deviation (SD). To generate the standard deviation curves the same general equation is used, but the $\mathrm{k}$ constant is replaced by a $\mathrm{k}+\mathrm{SD}$ or $\mathrm{k}-\mathrm{SD}$. The range between the SD curves tends to cover other model prediction. According to the authors, the range within the SD could predict the fatigue property of $50 \%$ of the population making it a strong asset to DHM software.

\section{(1) $\operatorname{Tcem}(t)=\operatorname{Tmax} e^{d t}-k \frac{T \log d t}{T \max } \Delta t$}

Table 1. Parameters in joint fatigue and recovery model

\begin{tabular}{lll}
\hline Item & Unit & Description \\
\hline Tcem $(\mathrm{t})$ & $\mathrm{Nm}$ & Joint moment capacity at time instant $\mathrm{t}$ \\
Tmax & $\mathrm{Nm}$ & Maximum joint moment \\
$\mathrm{k}$ & $\mathrm{min}^{-1}$ & Fatigue ratio constant value (joint specific) \\
Tload $(\mathrm{t})$ & $\mathrm{Nm}$ & Joint load moment at time instant $\mathrm{t}$ \\
$\mathrm{t}$ & $\mathrm{min}$ & Time \\
$\mathrm{R}$ & $\mathrm{min}^{-1}$ & Recovery ratio constant \\
\hline
\end{tabular}

Table 2. Constants k used for experimental validation. (source: Ma and Al., 2011)

\begin{tabular}{llll}
\hline Joint & $\mathrm{k}$ & $\begin{array}{l}\mathrm{k}^{-} \\
\text {(inferior SD limit) }\end{array}$ & $\begin{array}{l}\mathrm{k}^{+} \\
\text {(superior SD limit) }\end{array}$ \\
\hline Elbow & 1.1616 & 0.7881 & 2.2075 \\
Shoulder & 1.3224 & 0.8397 & 3.1104 \\
\hline
\end{tabular}

Ma and al. (2010) also proposed a recovery model that takes into account the level of fatigue at the beginning of the recovery phase, time and maximum joint moment. This recovery model is consistent with the previous fatigue model. The recovery equation, Eq. (2) uses the same parameters apart from the $\mathrm{k}$ constant which is replaced by an $\mathrm{R}$ parameter to describe the rate of recovery.

\section{(2) $\operatorname{Tem}(t+\Delta \mathrm{t})=\operatorname{Tmax}+(\operatorname{Tcem}(t)-T \max ) e^{-R \Delta t}$}

Frey Law and Avin (2010) developed a jointspecific fatigue model based on a meta-analysis of several static experiments. A total of 194 experiments and 369 data points were used for the ankle, elbow, hand, knee, shoulder, trunk and wrist. To demonstrate the relationship between intensity and ET, the power model provided the best fit to the experimental data (see figure 1). This study has shown significant ET differences between joints and concluded that a single generalized ET model does not adequately represent fatigue across joints. For example, the ankle is a lot more fatigue resistant than the shoulder. As a result, one power curve per joint was produced.

Joint specific endurance has been observed in several studies. El ahrache (2005) explored the difference between upper body and back/hip endurance. The conclusions were showing significant differences between muscles of these two body parts. Upper limbs ET was significantly lower than back/hip. Frey Law and Avin (2010) also explored the differences between joint endurance. As stated, "The ankle was the most fatigue-resistant, followed by the trunk, hand/grip, elbow, knee and finally the shoulder was most fatigable". This is in accordance with Ma's specific joint constants. These studies show that a single whole body fatigue prediction model cannot provide precise information on joint specific fatigue levels. An accurate model would tend to be joint specific. For similar situations, several studies had measured different MET. To be inclusive, a model would define a range of MET values in function of the intensity. Implemented into DHM software, such tool could help practitioners prevent overexertion and, therefore, risks of musculoskeletal disorders. Even if this is not aimed for a specific individual, a model that provides a MET prediction for a targeted population helps to identify potentially dangerous tasks and defining an efficient work organization.

\section{Chosen Model}

After comparison of different models in the above literature review, the decision was made to use joint level extended Ma's fatigue model. This MET prediction model was chosen because of the simplification of its application. It can also be individual specific, because it includes the maximum voluntary contraction factor. The general equation becomes joint specific by changing only the constants value. It includes the intensity of the effort, the time of exertion as well as the relative accumulated fatigue, which are risk factors of developing MSD's. All the required input parameters can be provided using the Dassault Systemes Virtual Ergonomics Solution DHM tool. 
Ma's model also provides SD information. The standard deviation is an interesting information for a fatigue assessment tool because MET can vary a lot from an individual to another due to internal factors such as genetic, motivation or general health condition. Therefore, a single mean MET curve cannot assess fatigue level adequately, especially at low intensity. The addition of SD curves can increase the usability of a fatigue prediction tool. As opposed to a single mean prediction curve, the SD curves provide a range that allows to relativize the predicted fatigue, especially for lower intensity tasks.

To keep consistency Ma's recovery model has also been used. According to the authors, the recovery rate from fatigue muscle motor unit is assumed to be constant, not joint-specific (Liu et al. 2002, Wood et al. 1997). The $\mathrm{R}$ constant value has been defined to 2.4 .

\section{Methods}

\subsection{Validation with published static MET results}

An extensive review of static fatigue experiments has been made in order to compare the results with Ma's model. Frey Law and Avin meta-analysis (2010) was used in the early stage of the research to find MET experiments. More studies were found and selected following inclusion criteria: Static contraction exerted at a certain percentage of maximal voluntary contraction (\%MVC); Healthy subjects; Effort exerted until failure (Endurance Time); Single joint implication. The shoulder and elbow joints were selected for the first phase of comparison. A total of 8 experiments have been chosen for the shoulder joint, and 37 for the elbow. The maximum endurance time (MET) was then extracted from the selected studies for a total of 35 values represented by points on the shoulder results graphic (figure 3) and 171 points for the elbow results graphic (figure 4). A comparison of MET for different intensities of exertion has been made using Ma's extended model. Note that the data collected among experiments included men and women, people of different ages as well as different angles of exertion. The validation has only been made for intensities between $10 \%$ and $80 \%$ of MVC. Intensities below $10 \%$ were excluded due to a restricted amount of experiments and large interindividuals experiments found in literature. Few industrial tasks where requiring an exertion over $80 \%$ of MVC in a static position

\subsection{Laboratory task simulation}

Since this is an exploratory study, only two experiments, each executed by a different subject have been done to verify if Ma's extended model (2010) gives adequate results. Both experiments had the same protocol, apart from the time of effort/recovery. The experiments consisted in lifting a box to move it from one shelf to another in a shoulder flexion movement. Subjects were only allowed to use the arms in the movement and were instructed to keep the rest of the body still. A milk crate with a total of $3.64 \mathrm{Kg}$ was used. Two shelves were placed at the height of $95 \mathrm{~cm}$ and $165 \mathrm{~cm}$. A metronome was used to make sure subject's rhythm was slow and controlled. Subjects were asked to perform task until exhaustion in order to obtain a MET.

The task could end either by voluntary withdrawal or by incapacity to maintain the prescribed rhythm. Both subjects were 27 years old, healthy and without any history of upper body disorders. A maximum shoulder voluntary contraction (MVC) test was done lifting a dynamometer with both hands. For the MVC measurement, the subjects were asked to develop maximum joint moment in a 90 degree shoulder flexion position while the dynamometer was attached to the ground. The best result out of three trials was considered to be the subject maximal strength and then has been converted into joint moment.

\subsubsection{Experiment 1}

The task consisted of a continuous effort, without recovery period and until exhaustion. The shoulder work load of the task was between 9 to $19 \%$ of the subject maximal capacity. The rhythm of execution had to be slow and controlled, without marked acceleration. Five movements/minute (up and down) were executed.

\subsubsection{Experiment 2}

The task consisted of an intermittent effort divided as such: 8 minutes of effort / 3 minutes static recovery (arm along body) / effort until exhaustion. The shoulder work load of the task was ranged between 12 to $25 \%$ of the subject maximal capacity. The rhythm of execution had to be slow and controlled, without 
marked acceleration. Five movements/minute (up and down) were executed.

To simulate the task, 5 key postures have been reproduced in the Dassault Systemes DHM software, as shown in figure 2. Shoulder moments $(\mathrm{Nm})$ were extracted from each key posture and introduced into the Ma's dynamical extended model. Time of movement was also included.

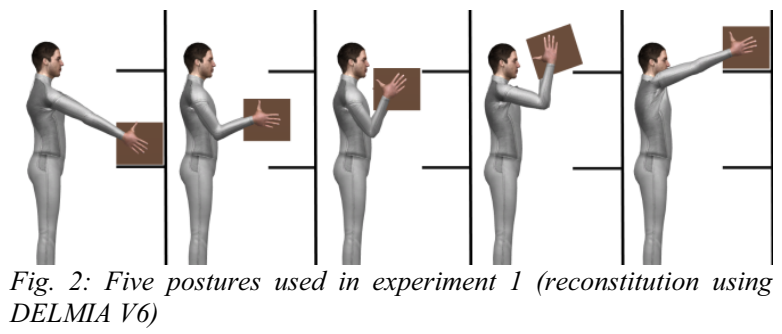

\section{Results}

The first test to assess the model is based only on data coming from the literature. It consists in verifying if the MET values found in the extensive review of static fatigue experiments are close to the Ma's prediction curve and fit within the standard deviation curves. To organize the results, all MET found were grouped by studied joint. Figure 3 shows the MET found for the shoulder and figure 4 does it for the elbow. In both chart, the solid line shows the mean fatigue prediction of the model and the dashed lines delimits the zone defined by the standard deviation. Each dot represents a MET value from static experiments found in the literature. In general, the comparison between static experiments and Ma's modified dynamical model shows a similar tendency for both shoulder and elbow joints. The variation between experiments increases with a smaller intensity of exertion (\%MVC). The standard deviation curves also show that the variability is increasing with the diminution of intensity. All 35 shoulder experimental data points fit within Ma's fatigue prediction range. However, with the elbow, several results are outside the prediction zone.

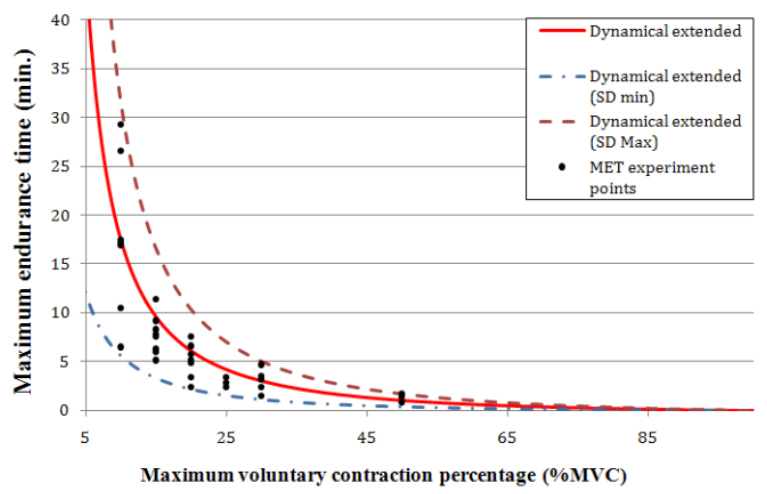

Fig. 3: Comparison between Ma's dynamical extended model (2009) and several static maximum endurance time experiments for the shoulder joint.

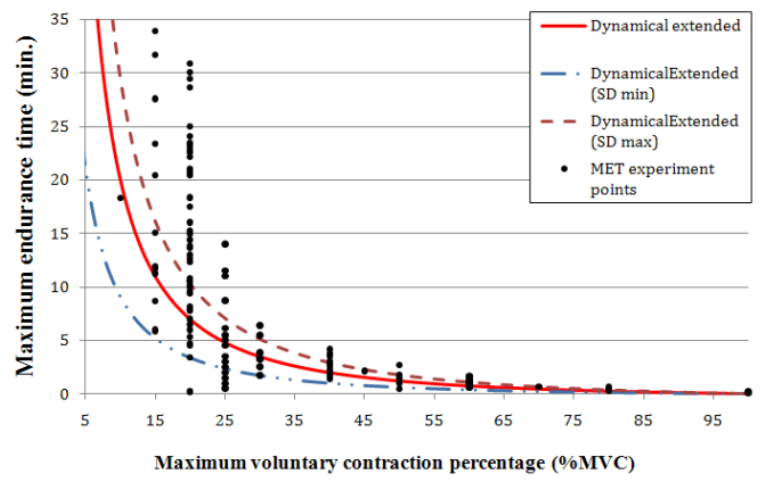

Fig. 4: Comparison between Ma's dynamical extended model (2009) and several static maximum endurance time experiments for the elbow joint.

Figure 5 shows the shoulder predicted fatigue for the experiment 1 (solid line) and the zone delimited by the standard deviation (dashed lines). The calculation sampling is 60 values per minute according to the task phases and execution rhythm. The fatigue accumulation continuously reduces the remaining capacity, since this task has no recuperation phase. In theory, the subject should drop out of the task when his remaining capacity reaches the task effort requirement (shown by the small dashed line). To simplify the failure curve and to ease the reading of the chart, the failure level has been established as the maximum joint moment required executing the task (worst case scenario). The predicted failure time is then the reach of the failure curve with the remaining capacity curve. The results show that the model predicts a failure time around 9 minutes while the subject fails at $7 \mathrm{~min} .45 \mathrm{sec}$. However, if the standard deviation (from 4 to $15 \mathrm{~min}$.) is considered, the subject failure time is within the predicted failure time range. 


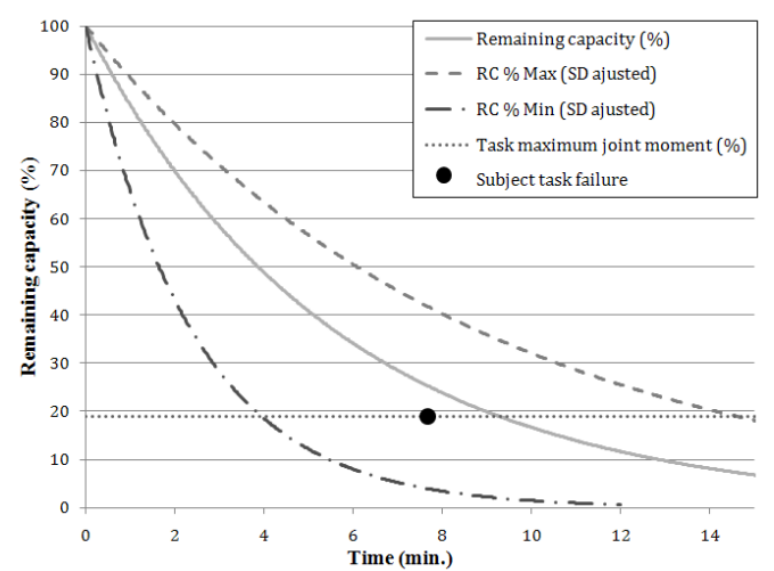

Fig. 5: Task simulation (experiment 1) using Ma's extended model (2009) for shoulder joint.

The second experiment (see figure 6) differs from the first one by the presence of a 3 minutes recovery break in the middle of the simulation (at $8 \mathrm{~min}$.). By considering only the remaining capacity curve, the model would have predicted a failure at 6 minutes, but test subject's measured endurance time is $21 \mathrm{~min} 5 \mathrm{sec}$. The recovery allows the predicted remaining capacity to get back over the failure level. Its 3 minutes duration also seems to allow a full recovery, which resets the inter-subject variability. A large part of the zone delimited by the standard deviation curve is located under the failure level.

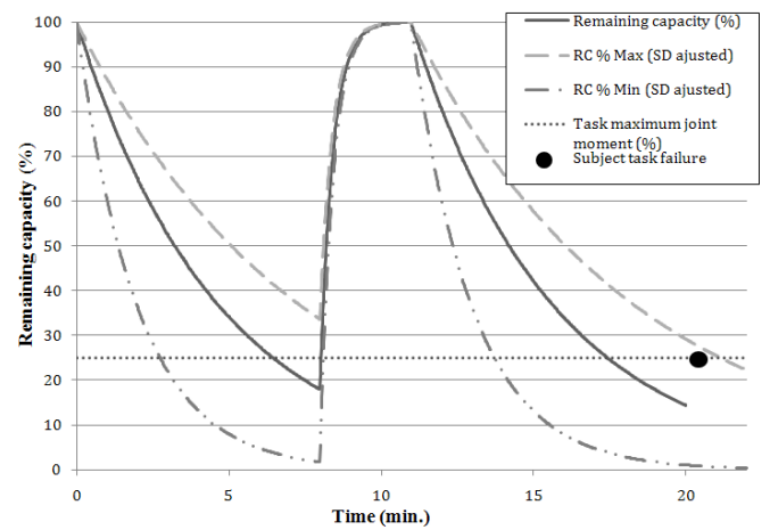

Fig. 6: Task simulation (experiment 2) using Ma's extended model (2009) for shoulder joint.

\section{Discussion}

Results for both static experiment data validation and dynamic subject task simulations were satisfying. On the static side, all 35 shoulder static MET experiment results fit within Ma's dynamical extended model range. Figures 3 and 4 show a greater difference between studies results when the work intensity is lower. Elbow MET static experiment results (Figure 4) tend to have a larger variation. Therefore, Ma's model does not cover the totality of the MET values. Between 15 and $25 \%$ of MVC, many experiments results aren't included within Ma's model SD. These results are in accordance with many other studies (Matiassen and Winkel, 1992; Gerdle and al., 1993). Fallentin (1991) reports a coefficient of variation in the order of $50 \%$ and for a $10 \%$ of MVC bent. The variation can be explained by methodological aspect as so by physiological. For example, the way the MVC is assessed can have an important impact on the result. An experimental subject can obtain several different results depending of its motivation, fear, the number of trial, etc. The tension developed by a muscle is impacted by its length, so is the posture. Moreover, the segment mass should be considered as a load and justify the use of joint moment, which is more standardized than a force in Newton. On the physiological side, the difference of fatigability between joints as much as the interindividual variability is related to the muscle constitution. The most commonly stated in literature is that the ratio of type 1 and type 2 fibers present in the active muscles group is different for each joint. An effort below $30 \%$ of the capacity uses type 1 fibers (Bigland Ritchie and Woods, 1984) which are the most fatigue resistant. An individual with a greater amount of type 1 (slow twitch) muscle fibers would have a better fatigue resistance. A small difference in the slow twitch ratio would have an important effect on the MET result. Above 30\%, the MET is considerably reduced, therefore the variability is reduced.

MET predictions for low intensity of exertion tend to be more challenging. The addition of standard deviation curves to Ma's prediction provides a wider range of possible MET for low intensities. It follows the tendency of getting a larger inter-individual variation of MET results for low percentage of MVC. If Ma's model does not cover all literature results, it tends to cover it for the elbow. Even if that coverage is not total, in an ergonomics perspective, it is preferable to underestimate fatigue levels than the opposite.

Both failure times determined in the task simulations with real subject fit in the predictions done with Ma's model and the virtual simulation data. The experiment 1 result (continuous effort) is slightly 
below the mean value of the curve. Experiment 2 result (intermittent effort) is closer to the higher end of the range. Since the simulated task requires an effort lower than $25 \%$ of the MVC, the variability is also observed in these two experiments.

Psychological factors can also influence MET. Experiments conducted until exhaustion can be very difficult to handle for certain people. The pain tolerance threshold can vary from a subject to another. Therefore, it has a huge influence on experimental results. Because this fact also applies to the MVC measurement, the maximal joint moment has been measured for each subjects used in the laboratory experimentations. However, this individual specific information may not be available when the human factors expert uses a prediction tool. In such case, DHM software can refer to scientific documentation to define the population MVC value. Considering this value represents the average of a population, the curves may tend to get lower and more conservative.

Because of this variability, it is important for the human factors expert to visualize the prediction graphically and especially the standard deviation curves. Most of the time, such fatigue prediction tool cannot provide a "pass/fail" answer. Its use should be seen as an indicator to estimate the effort level and the proportion of the population that may execute the task.

\section{Limits}

Other factors could also have an influence on muscular fatigue. However, they were excluded from our analysis due to time limitations. For example, several external factors such as temperature or relative humidity can have an influence on the capacity to sustain muscular contraction. Internal factors such as age, sex or level of physical activity can also influence a person's fatigue resistance and measured maximal joint moment developed.

The DHM software common user may not have the maximal joint moment for specific individual. In such case he would have to refer to a maximal mean value generally set into the software. The fatigue prediction results have to be interpreted cautiously, keeping in mind the large inter-individual variation, especially at low intensity.

At this point, the recovery model is only suitable for inactive recovery. The recovery is activated when the joint moment equals $0 \mathrm{Nm}$. Otherwise, fatigue would accumulate, even for values as low as $1 \%$. This is the case for very low joint moment that may result from a not optimal posture creation. To be more representative of the reality, an active recovery should be added for low intensity effort. Further work will be necessary to adapt the recovery model for active recovery.

So far, the extended MET model has only been tested for values between $15 \%$ and $99 \%$ of maximum voluntary contraction. More task simulations and more subjects would be needed to solidify the validation of Ma's model. Validation would also be needed for other joints.

\section{Conclusion}

In conclusion, based on elbow and shoulder joint, Ma's extended model has been compared to published static MET experiments. It has also been compared to two laboratory task simulations. Ma's model is capable of covering most of MET static experiments by including standard deviation curves in order to create a range of possible values. MET results from single subject task simulations also fits within Ma's model range. The fatigue prediction standard deviation curves can be trusted.

Implemented in DHM software, the graphical representation of the fatigue prediction, as presented for the simulation task, could help practitioners evaluate the risk associated to fatigue. Instead of getting a single time to task exertion failure value, the human factors expert can benefit of viewing graphically the predicted fatigue pattern and the SD of a simulated task and use it as a guideline. That gives the human factors expert the possibility to nuance the results in consideration of other environment factors and some realities specific to the industry.

In order to do a better validation of the $\mathrm{k}$ parameter, further experiments must be done by simulating more tasks in a laboratory. As for now, only shoulder and elbow joints have been tested and more subjects will be necessary for further validation. To compare laboratory task execution and the prediction model they should have more comparison element than the failure time. A new methodology using electromyography is actually in process. Further market analysis is needed to determine if such tool 
implementation would bring added value to DHM users.

\section{References}

Alizadehkhaiyat, O., Fisher, A., Kemp, G., Frostick, S., 2007. Strength and fatigability of selected muscles in upper limb: Assessing muscle imbalance relevant to tennis elbow. Journal of Electromyography and Kinesiology, 17, 428-436

Armstrong, T., Buckle, P., Fine, L.J., Hagberg, M., Jonsson, B., Kilborn, A., Silverstein, B.A., Sjogaard, G., Viikari-Juntura, 1993. A conceptual model for work-related neck and upperlimb musculoskeletal disorders. Scandinavian Journal of Work, Environment and Health 19, 73-84.

Baudry, S., et al., 2009. Load type influences motor unit recruitment in biceps brachii during a sustained contraction. Journal of Neurophysiolgy, 102 (3), 1725-1735.

Bazzucchi, I., et al., 2005. Differences in the forcelendurance relationship between young and older men. European Journal of Applied Physiology, 93 (4), 390-397.

Bertmaring, I., Babski-Reeves, K., and Nussbaum, M., 2008. Infrared imaging of the anterior deltoid during overhead static exertions. Ergonomics, 51 (10), 1606-1619.

Bigland-Ritchie, B. and Woods, JJ, 1984. Changes in muscle contractile properties and neural control during human muscular fatigue. Muscle Nerve 7: 691-699,

Bongers, P.M., De Winter, C.R., Kompier, M.A.J., Hildebrandt, V., 1993. Psychosocial factors at work and musculoskeletal disorders. Scandinavian Journal of Work, Environment and Health 19, 297-312.

Booth, F.W. and Thomason, D.B., 1991. Molecular and cellular adaptation of muscle in response to exercise: Perspectives of various models. Physiology Review, 71(2), 541-585.

Burdorf, A., 1992. Exposure assessment of risk factors for disorders of the back in occupational epidemiology. Scandinavian Journal of Work, Environment and Health 18, $1-9$.

Calder, K.M., Stashuk, D.W., and Mclean, L., 2008. Physiological characteristics of motor units in the brachioradialis muscle across fatiguing low-level isometric contractions. Journal of Electromyography \& Kinesiology, $18(1), 2-15$.

Chaffin, D. B., Digital Human Modeling for Vehicle and Workplace Design, SAE Internationnal, Pensilvania, 2001, 202 pages.

Chaffin, D. B., Andersson G. B. J., Martin B. J. Occupational biomechanics, 3rd edn.Wiley-Interscience, 1999, 600 pages.

Cogiamanian, F., et al., 2007. Improved isometric force endurance after transcranial direct current stimulation over the human motor cortical areas. European Journal of Neuroscience, 26 (1), 242-249.
Dimitrova, N.A., et al., 2009. Fatigue analysis of interference emg signals obtained from biceps brachii during isometric voluntary contraction at various force levels. Journal of Electromyography \& Kinesiology, 19 (2), 252-258.

Edwards, R.H., 1981. Human muscle function and fatigue. Ciba Foundation Symposium, 82, 1-18.

El ahrache, K., Imbeau, D., Farbos, B., 2005. Percentile values for determining maximum endurance times for static muscular work. International Journal of Industrial Ergonomics 36 (2), 99-108.

Fallentin, N. and Jorgensen, K., 1992. Blood pressure response to low level static contractions. European Journal of Applied Physiology and Occupational Physiology, 64 (5), 455-459.

Fallentin, N., 1991. Cardiovascular adjustments to static exercise: neural control mechanisms, muscle fiber composition and electromyographic activity, Doctoral dissertation, August Krogh Institute, University of Copenhagen, Denmark.

Felici, F., et al., 2001. Effect of human exposure to altitude on muscle endurance during isometric contractions. European Journal of Applied Physiology, 85(6), 507-512.

Frey Law, L., Avin, K., 2010. Endurance time is joint-specific: a modelling and meta-analysis investigation. Ergonomics. Jan;53(1):109-29.

Gerdle, B., Edstrom, M., and Rahm, M., 1993. Fatigue in the shoulder muscles during static work at two different torque levels. Clinical Physiology, 13 (5), 469-482.

Hansen, J.W., 1967. Effect of dynamic training on the isometric endurance of the elbow flexors. Internationale Zeitschrift fur Angewandte Physiologie, einschliesslich Arbeitsphysiologie, 23 (4), 367-370

Hendrix, C.R., et al., 2009b. A comparison of critical force and electromyographic fatigue threshold for isometric muscle actions of the forearm flexors. European Journal of Applied Physiology, 105 (3), 333-342.

Hermans, V. and Spaepen, A.J., 1997. Muscular activity of the shoulder and neck region during sustained and intermittent exercise. Clinical Physiology, 17 (1), 95-104.

Hoeger Bement, et al., 2009a. The role of the menstrual cycle phase in pain perception before and after an isometric fatiguing contraction. European Journal of Applied Physiology, 106 (1), 105-112.

Hunter, S.K., et al., 2004b. Fatigability of the elbow flexor muscles for a sustained sub maximal contraction is similar in men and women matched for strength. Journal of Applied Physiology, 96 (1), 195-202.

Hunter, S.K., et al., 2003. Activation among the elbow flexor muscles differs when maintaining arm position during a fatiguing contraction. Journal of Applied Physiology, 94 (6), 2439-2447. 
Hunter, S.K., et al., 2002. Task differences with the same load torque alter the endurance time of sub maximal fatiguing contractions in humans. Journal of Neurophysiology,88 (6), 3087-3096.

Hunter, S.K., Critchlow, A., and Enoka, R.M., 2005a. Muscle endurance is greater for old men compared with strengthmatched young men. Journal of Applied Physiology, 99 (3), 890-897.

Hunter, S.K., Critchlow, A., and Enoka, R.M., 2004a. Influence of aging on sex differences in muscle fatigability. Journal of Applied Physiology, 97 (5), 1723-1732.

Hunter, S.K. and Enoka, R.M., 2003. Changes in muscle activation can prolong the endurance time of a sub maximal isometric contraction in humans. Journal of

Applied Physiology, 94 (1), 108-118.

Hunter, S.K. and Enoka, R.M., 2001. Sex differences in the fatigability of arm muscles depend on absolute force during isometric contractions. Journal of Applied Physiology, 91 (6), 2686-2694.

Kilbom, A., et al., 1983. Physiological and psychological indices of fatigue during static contractions. European Journal of Applied Physiology and Occupational Physiology, 50 (2), 179-193.

Klass, M., et al., 2008. Spinal mechanisms contribute to differences in the time to failure of sub maximal fatiguing contractions performed with different loads. Journal of Neurophysiology, 99 (3), 1096-1104.

Kleine, B.U., et al., 2000. Surface emg mapping of the human trapeziums muscle: The topography of monopolar and bipolar surface emg amplitude and spectrum parameters at varied forces and in fatigue. Clinical Neurophysiology, 111 (4), 686-693.

Krogh-Lund, C. and Jorgensen, K., 1993. Myo-electric fatigue manifestations revisited: Power spectrum, conduction velocity, and amplitude of human elbow flexor muscles during isolated and repetitive endurance contractions at 30\% maximal voluntary contraction. European Journal of Applied Physiology and Occupational Physiology, 66 (2), 161-173.

Krogh-Lund, C. and Jorgensen, K., 1992. Modification of myoelectric power spectrum in fatigue from $15 \%$ maximal voluntary contraction of human elbow flexor muscles, to limit of endurance: Reflection of conduction velocity variation and/or centrally mediated mechanisms? European Journal of Applied Physiology and Occupational Physiology, 64 (4), 359-370.

Liu, J.Z., Brown, R.W., Yue, G.H., 2002. A dynamical model of muscle activation, fatigue, and recovery. Biophysical Journal 82, 2344-2359.

Lowery, M., Nolan, P., and O'malley, M., 2002. Electromyogram median frequency, spectral compression and muscle fibre conduction velocity during sustained submaximal contraction of the brachioradialis muscle. Journal of Electromyography and Kinesiology, 12 (2), 111-118.
Ma, L., and Al., 2011. A novel approach for determining fatigue resistances of different muscle groups in static cases. International Journal of Industrial Ergonomics 41, 10-18

Ma, L., and Al., 2010. A new muscle fatigue and recovery model and its ergonomics application in human simulation. Virtual and Physical Prototyping, 5 (3),123-137

Ma, L., and Al., 2009. A new simple dynamic muscle fatigue model and its validation. International Journal of Industrial Ergonomics 39, 211-220

Mamaghani, N.K., et al., 2001. Changes in surface emg and acoustic myogram parameters during static fatiguing contractions until exhaustion: Influence of elbow joint angles. Journal of Physiological Anthropology and Applied Human Science, 20 (2), 131-140.

Mathiassen, S.E. and Ahsberg, E., 1999. Prediction of shoulder flexion endurance from personal factors. International Journal of Industrial Ergonomics, 24, 315-329.

Mathiassen, S.E. Winkel, J., 1992. Can occupational guidelines for work-rest schedules be based on endurance time data? Ergonomics 35, 253-259.

Mottram, C.J., et al., 2006. Time to task failure varies with the gain of the feedback signal for women, but not for men. Experimental Brain Research, 174 (3), 575-587.

Nicolas, A., et al., 2008. The influence of circadian rhythm during a sustained sub maximal exercise and on recovery process. Journal of Electromyography and Kinesiology, 18 (2), 284-290.

Ohashi, J., 1993. Effects of contraction level on the changes of surface electromyogram during fatiguing static contractions. Annals of Physiology Anthropolgy, 12 (4), 229-241.

Orizio, C., et al., 1992. Muscle sound and electromyogram spectrum analysis during exhausting contractions in man. European Journal of Applied Physiology and Occupational Physiology, 65 (1), 1-7.

Riley, Z.A., Baudry, S., and Enoka, R.M., 2008. Reflex inhibition in human biceps brachii decreases with practice of a fatiguing contraction. Journal of Neurophysiology, 100 (5), 2843-2851.

Roe, C., et al., 2006. Long-term repeatability of force, endurance time and muscle activity during isometric contractions. Journal of Electromyography and Kinesiology, 16 (1), 103-113.

Rohmert, W., 1960. Ermittlung von Erholungspausen fur statische Arbeit des Menschen. European Journal of Applied Physiology 18 (2), 123-164.

Rudroff, T., et al., 2007a. Accessory muscle activity contributes to the variation in time to task failure for different arm postures and loads. Journal of Applied Physiology, 102 (3), 1000-1006. 
Rudroff, T., et al., 2005. Net excitation of the motor unit pool varies with load type during fatiguing contractions. Muscle and Nerve, 31 (1), 78-87.

Rudroff, T., Staudenmann, D., and Enoka, R.M., 2008. Electromyography measures of muscle activation and changes in muscle architecture of human elbow flexors during fatiguing contractions. Journal of Applied Physiology, 104 (6), 1720-1726.

Sacco, P., et al., 1999. Corticomotor excitability and perception of effort during sustained exercise in the chronic fatigue syndrome. Clinical Neurophysiology, 110 (11), 18831891.

Sato, H., et al., 1984. Endurance time and fatigue in static contractions. Journal of Human Ergology (Tokyo), 13(2), $147-154$.

Schulte, E., et al., 2004. Experimental muscle pain increases trapeziums muscle activity during sustained isometric contractions of arm muscles. Clinical Neurophysiology, 115 (8), $1767-1778$

Semmler, J.G., Kutzscher, D.V., and Enoka, R.M., 1999. Gender differences in the fatigability of human skeletal muscle. Journal of Neurophysiology, 82 (6), 3590-3593.

Sjogaard, G., 1986. Intramuscular changes during long-term contraction. In: N. Corlett, J. Wilson, and I. Manenica, eds. The ergonomics of working postures. Londres: Taylor and Francis, 136-143.

Staudenmann, D., Rudroff, T., and Enoka, R.M., 2009. Pronation-supination torque and associated electromyography activity varies during a sustained elbow flexor contraction but does not influence the time to task failure. Muscle and Nerve, 40 (2), 231-239.

United States Department of Labor, 2008. Definition of "Musculoskeletal Disorders" by the Bureau of Labor Statistics, http://www.bls.gov/

Wood, D., Fisher, D., Andres, R., 1997. Minimizing fatigue during repetitive jobs: optimal work-rest schedules. Human Factors: The Journal of the Human Factors and Ergonomics Society 39 (1), 83-101.

Yassierli and Nussbaum, M.A., 2008. Utility of traditional and alternative emg-based measures of fatigue during lowmoderate level isometric efforts. Journal of Electromyography and Kinesiology, 18 (1), 44-53.

Yoon, T., et al., 2007. Mechanisms of fatigue differ after low and high-force fatiguing contractions in men and women. Muscle and Nerve, 36 (4), 515-524.

Yoon, T., et al., 2008. Age-related muscle fatigue after a lowforce fatiguing contraction is explained by central fatigue. Muscle and Nerve, 37 (4), 457-466. 